\title{
1 Effect of Gelation on the Colloidal Deposition of 2 Cellulose Nanocrystal Films \\ 3
}

4

Alican Gençer, ${ }^{a}$ Jonas Van Rie, ${ }^{a}$ Salvatore Lombardo, ${ }^{a}$ Kyongok Kang, ${ }^{b}$ Wim Thielemans ${ }^{\mathrm{a},}{ }^{,}$

${ }^{a}$ Renewable Materials and Nanotechnology Research Group, Department of Chemical Engineering, KU Leuven, Kulak Kortrijk Campus, Etienne Sabbelaan 53, 8500 Kortrijk, Belgium

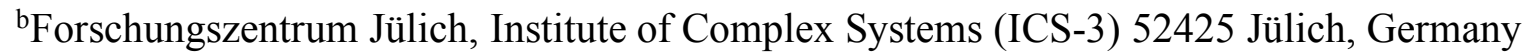

*Corresponding author: Wim.Thielemans@kuleuven.be

\section{ABSTRACT}

One of the most important aspects in controlling colloidal deposition is manipulating the homogeneity of the deposit by avoiding the coffee-ring effect caused by capillary flow inside the droplet during drying. After our previous work where we achieved homogeneous deposition of cellulose nanocrystals (CNCs) from a colloidal suspension by reinforcing Marangoni flow over the internal capillary flow ${ }^{1}$, we now set out to reduce the importance of capillary flow inside a drying droplet by inducing gelation. In this paper, we discuss the effect of gelation on the deposition pattern and on the self-assembly of CNCs during droplet drying. CNC films were obtained by drop casting $\mathrm{CNC}$ suspensions containing $\mathrm{NaCl}$ and $\mathrm{CaCl}_{2}$ salts. A mixed methodology using rheological and depolarized dynamic light scattering was applied to understand the colloidal behavior of the CNCs. In addition, analysis of the mixture's surface tension, viscosity, and yield stress of the suspensions were used to gain deeper insights into the deposition process. Finally, the understanding of the gelation behavior in the drying droplet was used to exert control over the deposit where the coffee-ring deposit can be converted to a dome-shaped deposit. 


\section{INTRODUCTION}

Periodical arrangement of particles can lead to the generation of structural colors without the use of dyes or colorants. This so-called structural color formation is observed in nature for many animals and plants. ${ }^{2,3,4,5,6}$ Mimicking nature, films showing iridescence or color can be produced by carefully ordering nanoparticles. One straightforward way of achieving this is to make use of self-assembling colloidal nanoparticles that can achieve long-range order. For example, ribbon-like cellulose nanocrystals ( $\mathrm{CNCs}$ ) have been shown to produce optically active films. ${ }^{7,8,9,10} \mathrm{CNCs}$ self-assemble in suspension above a critical concentration into chiral nematic structures. ${ }^{9,11}$ This long range order can be retained in dried films leading to color formation associated with the chiral nematic order of the CNCs and the associated photonic band gap.

The effects of additional components mixed with CNCs has also been investigated for polymers $^{12,13,14,15}$ or salts ${ }^{16,17,18}$ to understand how these additives affect the CNC self-assembly in suspension and/or in deposited films. Furthermore, the aggregation ${ }^{19}$ and the gelation ${ }^{20,21,22}$ of CNCs with changes in ionic strength of $\mathrm{CNC}$ suspensions have also been investigated using SANS, ${ }^{19}$ SAXS,${ }^{20}$ and Monte Carlo simulations.${ }^{23}$ Increasing the charge and ionic radius of the added cations have further been shown to increase the elastic moduli of the gels, ${ }^{20,24}$ which was attributed to fact that mesh size of the network was changed relative to the ions added to the system. In addition, the gelation kinetics and the gel structure of $\mathrm{CNCs}$ have been studied in the literature using confocal laser scanning microscopy and light scattering. ${ }^{25}$ Gelation and aggregation kinetics will be important in depositing CNC suspension with added salt as concentrations will change during water evaporation and this will affect deposition homogeneity and CNC structuring. Depending on conditions, long range order in the CNCs can be preserved or lost. 
Drop-casting of colloidal suspensions followed by solvent evaporation is one of the experimental approaches to study colloidal self-assembly processes and to obtain $\mathrm{CNC}$ films. ${ }^{26,27,28,29}$ However, droplet drying normally leads to non-uniform deposits due to the coffee-ring effect, ${ }^{30}$ i.e. the preferential deposition of particles at the outer edges of the drying drop. This complicates the analysis of the self-assembly as inhomogeneity is introduced in the deposit. The inhomogeneous character of the deposit is also non-desirable for many applications. The increased deposition of particles at the edge is caused by capillary flow toward the edge during drying due to the replenishment of water as the solvent evaporation rate is higher at the droplet edge. This phenomenon can be offset by manipulation of the internal droplet flow via generation of thermal Marangoni flow, ${ }^{31}$ surfactant-induced Marangoni flow, ${ }^{32,33}$ solutal Marangoni flow, ${ }^{1,34}$ and light-induced Marangoni flow ${ }^{35}$. Marangoni flow is an internal flow from the drying droplet edge inwards along the droplet surface that counteracts the capillary flow to avoid particle accumulation at the edge of the drying droplet. We have previously studied the structure formation and the deposition pattern of CNCs upon drying of droplets of CNC suspensions and described the effect of the flow field inside the drying droplet on the deposition patterns of the CNCs. ${ }^{1}$ The effect of various parameters such as colloidal stability, concentration, atmosphere, and deposition profile on the optical properties of deposited films were elucidated. ${ }^{1}$ We then controlled the relative ratio of Marangoni to capillary flow to regulate the deposition pattern and obtain a homogeneous deposit. ${ }^{1}$

Alternatively, alteration of the electrostatic particle-particle and particle-substrate interactions have also been used to get more uniform films. ${ }^{36,37}$ Another methodology towards uniform deposits is to make use of skin formation during drying. ${ }^{38}$ In this method, the particles are trapped at the air-liquid interface and they deposit directly as the solvent evaporates. A recent study of the deposition of suspended particles also indicated that at elevated temperatures the particles tend to kinetically accumulate at the air-liquid interface via rapid evaporation leading 
to a more uniform deposit. ${ }^{39}$ Colloidal gelation has also been shown to effectively control deposition as it suppresses radial flow. Gel formation will restrict the radial capillary flow and thus particle motion towards the drop edge. ${ }^{40} \mathrm{Gel}$ formation of colloidal CNCs has for example been used to produce antireflective coatings, by producing porous materials that reduce the refractive index of the supporting material. ${ }^{41,42}$ In another example, alignment of nanofibrils was achieve by using $\mathrm{Na}^{+}$ions to fix hydrodynamically aligned cellulose nanofibrils making use of the dispersion-gel transition. ${ }^{43}$ Non-aligned gelled CNC suspension could also be interesting as viscoelastic inks where they could be used for patterning $3 \mathrm{D}$ objects by direct ink writing. ${ }^{44}$ This has been shown in $3 \mathrm{D}$ printing of $\mathrm{CNC}$ particles suspended in water or a photopolymerizable monomer solution to produce 3D printed CNC based architectures. ${ }^{45,46}$ In order to have a control in extrusion and patterning processes, finite yield stresses are required for the suspensions and these could readily be achieved by salt-induced gelling of CNCs. It would thus be interesting to investigate whether salt addition to a drying drop can avoid the coffee ring effect and what the ensuing structuring of the CNCs is under those conditions.

Therefore, in this work, we extend on our previous work on drop deposition of CNC suspensions and report on the effects of salt addition on the deposition of CNC suspensions during droplet drying. The electrostatic interactions between CNCs were modulated by the addition of the electrolytes, leading to changes in viscosity and CNC diffusion rates. We carried out rheological measurements to understand network formation with the added salt while depolarized dynamic light scattering (DDLS) was used to extract the CNCs diffusion coefficients in the mixed systems. The effect of changes in diffusion rates and rheological properties were then used to describe the flow behavior inside the drying droplet and the ensuing deposition process and deposition profile. Finally, the effects on the optical properties of the films were characterized with cross-polarized light microscopy and linked to the deposition parameters. 


\section{EXPERIMENTAL SECTION}

\section{Materials}

Cotton wool and calcium chloride (96\%, granular) were purchased from Carl Roth GmbH. Sodium chloride (99.5\%) was purchased from Fisher Scientific. Sulfuric acid (95\%, RECTAPUR) was purchased from VWR International, while dialysis membranes (molecular weight cut-off of 12-14 kDa) were purchased from SpectrumLabs. Amberlite MB-6113 (for ion chromatography, mixed resin) was purchased from Acros Organics. Glass cover slides were purchased from Agar Scientific. All materials were used as received unless otherwise stated.

\section{Preparation of a Cellulose Nanocrystal (CNC) Suspension}

A cellulose nanocrystal suspension was obtained via sulfuric acid (10.06 M) hydrolysis of cotton wool over $40 \mathrm{~min}$ at $45{ }^{\circ} \mathrm{C}$ using the same procedure as used in our previous work. ${ }^{1}$ The obtained product was diluted with $1 \mathrm{~L}$ water and centrifuged 5 times at $10{ }^{\circ} \mathrm{C} 10000 \mathrm{rpm}$ with intermitted distilled water washes and decantation of the supernatant to remove excess acid. After centrifugation, samples were dialysed against deionized water for $48 \mathrm{~h}$ using a dialysis membrane (MWCO 12-14 kDa). The nanocrystal suspension was homogenized using a Branson Digital Sonifier, aliquots of $300 \mathrm{~mL}$ of suspension were sonicated for 3- minutes at amplitude $25 \%$ before filtration through a No. 2 fritted filter to remove remaining aggregates. The suspension was then mixed with Amberlite MB6113 resin to remove excess non- $\mathrm{H}^{+}$and $\mathrm{OH}^{-}$ions in solution. The resin was subsequently removed by filtration. The obtained initial aqueous suspension was then concentrated to the desired concentrations by evaporating excess water by rotary evaporation. The weight concentration of the cellulose nanocrystal suspensions was determined with thermogravimetric analysis. Full characterization of the CNCs can be found in ESI.

\section{Film Deposition}


The cellulose nanocrystal suspension was mixed with sodium chloride or calcium chloride to obtain the desired ionic strength prior to casting onto a dust-free glass slide. Glass cover slides were rinsed with isopropanol and dried with compressed air prior to use. Each film was prepared by depositing $10 \mu \mathrm{L}$ of a suspension. Drops were cast immediately after the $\mathrm{CNC}$ suspension was mixed with the desired salt to avoid gelation time effects (Figure SI 2, 4, 6), as it was noticed that leaving the suspension to equilibrate led to the formation of hydrogels at higher ionic strength (Figure SI 6) as expected from other literature reports. ${ }^{18,20,21}$ Images of drying droplets (ESI, Movie SI 1) were recorded in real time with a camera (DCC 3240C, Thorlabs) fitted with a zoom lens (MVL7000, Thorlabs) and analyzed with ImageJ to derive the droplet volume versus time using the same approach as used in our previous work. ${ }^{1}$ The deposited films were further characterized with a cross-polarized light microscope and by profilometry.

\section{Cross-Polarized Light Microscopy}

Cross-polarized light microscopy images were taken with an Olympus BX51 microscope. The glass slide was placed between crossed polarizers, and images were recorded using a digital colour CCD camera (Lumenera Infinity $2 \mathrm{Mpx}$ ).

\section{Profilometry}

Profilometry was used to determine the height profile of the deposited films through the center point of the deposited films. Profilometer traces were obtained with a Taylor-Hobson 120-L Form Talysurf using a diamond tip with a radius of $2.5 \mu \mathrm{m}$ and a measurement speed of $0.5 \mathrm{~mm} / \mathrm{s}$.

\section{Pendant Drop Tensiometry}

The surface tension of pendant drops containing different concentrations of CNCs was measured with a CAM200 (KSV NIMA) at room temperature. The measurement precision was determined using the Worthington number, Wo. ${ }^{47}$ The surface tension (Figure SI 1) 
determination of mixed systems becomes more difficult in case of the induced elasticity at the interface since pendant drop tensiometry depends on the balance between interfacial tension and gravitational forces. ${ }^{48}$ For such systems fitting accuracy was confirmed by the Bond number which was calculated to be $\sim 0.3$. The same setup CAM200 (KSV NIMA) was used for the determination of the initial contact angles of colloidal drops.

\section{Rheological Measurements}

An AR-G2 stress-controlled rotational rheometer (TA Instruments) was used to determine the rheological properties (viscosity changes and gelation) of the suspensions. All of the suspensions were tested at $20{ }^{\circ} \mathrm{C}$ using a double-wall concentric cylinder geometry with gap sizes of 380 and $420 \mu \mathrm{m}$. For each sample, the linear viscoelastic (LVE) limit was determined by carrying out an amplitude sweep experiment at a frequency of $1 \mathrm{~Hz}$. The LVE strain amplitude was used in the later frequency sweep experiments. Time sweep experiments were carried out at 5\% strain and a frequency of $1 \mathrm{~Hz}$. The yield stress values were obtained by fitting steady shear flow data with the Herschel-Bulkley model. ${ }^{49}$ In order to avoid shear induced reduction of the yield stress values of the mixtures, salt solutions were mixed with CNC suspensions which were already loaded into the geometry.

\section{Depolarized Dynamic Light Scattering (DDLS)}

DDLS measurements were carried out on an ALV 5000 autocorrelator to determine the scattered light electric field time autocorrelation function for each suspension. All measurements were carried out between 30-120 degrees with an angular step of 5 degrees for $300 \mathrm{~s}$. A toluene bath was used around the sample tubes to control the temperature and match the refractive index of the glass sample holder. The data was analyzed using the method of cumulants to extract the diffusion coefficients as described elsewhere. ${ }^{50}$ In short, the correlation function, $g_{l}(t)$, was fitted with Equation 1 to obtain the decay constant $\Gamma$, where $\Gamma_{V H, 1}=$ 
$1751 / \tau_{1}$ and $\Gamma_{V H, 2}=1 / \tau_{2}$, with $\tau_{i}$ the relaxation time for the slow mode $\left(\tau_{1}\right)$ and fast mode $\left(\tau_{2}\right)$ as 176 shown in Figure SI 12 . The obtained decay constant $\Gamma_{V H}$ was subsequently fitted with Equation 1772 to get the rotational $\left(\mathrm{D}_{\mathrm{R}}\right)$ and translational $\left(\mathrm{D}_{\mathrm{T}}\right)$ diffusion coefficients. ${ }^{51}$

$g_{1}(t)=A_{1} \exp \left(-\left(\Gamma_{V H, 1}\right) t\right)+A_{2} \exp \left(-\left(\Gamma_{V H, 2}\right) t\right)^{\beta}+B$

$\Gamma_{V H}=q^{2} D_{T}+6 D_{R}$

180

181

182

183

184

185

\section{RESULTS AND DISCUSSION}

Nanocrystals can form network structures and this occurs at lower concentrations as the ionic strength is increased. ${ }^{18,19,25,52}$ In this scenario, each nanocrystal acts as a collector, and nanocrystals will deposit onto each other due to screening of electrostatic repulsions. ${ }^{19,53}$ Full analysis of this behavior is very complex because of the shape of the cellulose nanocrystals, their longitudinal twist, and local surface charge information. Previously, molecular dynamics simulations were used to understand the structure and charge distribution of the cellulose nanocrystals with added $\mathrm{NaCl}^{54}$ An analysis of the 3D maps of the $\mathrm{Na}^{+}$density distribution confirmed that an increase in electrolyte concentration shrinks the electric interfacial layer and weakens the effective repulsion between charged $\mathrm{CNCs}^{54}$ Sodium ions have also been shown to screen the repulsive forces between negatively charged sulfate ester groups of the cellulose nanocrystals leading to network formation and gelation. ${ }^{19,20,23}$ The addition of $\mathrm{NaCl}$ and $\mathrm{CaCl}_{2}$ to the CNC suspension are both found to increase the viscosity and a yield stress is measured upon gelation. ${ }^{49,55}$ Other aqueous $\mathrm{CNC}$-cation interactions have also been studied by mixing CNCs with different salts resulting in the formation of hydrogels, ${ }^{20,52}$ where the formation of a network has been proven through rheological measurements. ${ }^{18,49}$

To describe the dynamic system of a drying droplet which contain anionic CNCs and cations, a finite element numerical model could be used to solve the Navier-Stokes, and the heat and 
mass transport equations in a Lagrangian framework. ${ }^{56,57}$ However, in studies of evaporating colloidal drops solving the diffusion equation of the solvent with boundary conditions depends on the particle concentration near the liquid interface. This leads to a complex moving boundary problem of nonlinear partial differential equations. ${ }^{58}$ Therefore, to avoid the complexity of full numerical simulations, dimensionless numbers can be introduced to understand the relative magnitudes of processes controlling the deposition of particles, and get a practical understanding of how to control the deposition pattern. ${ }^{1,59,60,61,62}$ For example, the capillary number is used to describe the relative effect of viscous forces to surface tension acting across an interface when characteristic fluid velocities are known. ${ }^{63}$ Through manipulation of the relative magnitudes of each of the contributions, it becomes possible to selectively reinforce a specific process and directly affect the colloidal deposition pattern, without having to carry out in-depth simulations of the drying process. ${ }^{1}$ In our study, we have taken this approach to understand the internal flow characteristics inside the drying drop. Using a modified capillary number, $C$ (Equation 3), we can compare the elastic modulus with the internal capillary pressure to quantify the elastic modulus at which capillary flow is completely stopped: ${ }^{40}$

$$
€=\frac{G^{\prime} r_{c}}{2 \gamma}
$$

The value for $C$ has to be higher than unity for the network (gel) integrity, expressed by the modulus, to resist the Laplace pressure inside the drying droplet, $P=2 \gamma / r_{c}$ with $r_{c}$ the radius of curvature and $\gamma$ the surface tension that is the driving force for capillary flow. To determine whether gelation upon salt addition is sufficient to limit the capillary flow in a drying droplet, the reason for the coffee-ring effect, we need to establish the magnitude of the gel yield stress at which capillary flow is inhibited. The elastic moduli of gels formed from aqueous CNC suspensions of 2 and $4 \mathrm{wt} \%$ by addition of $10 \mathrm{mM} \mathrm{NaCl}$ or $\mathrm{CaCl}_{2}$ to attain $\epsilon=1$ for a droplet with radius $2.5 \times 10^{-3} \mathrm{~m}$ (average radius for our experiments) are reported in Table 1 . All moduli 
fell in a similar range of several tens of Pa. Previous work noted that the actual yield stress value required to overcome capillary flow is lower than this calculated $G^{\prime}$ as there is a gradient in capillary pressure within the drying droplet. ${ }^{40}$ The determined $G^{\prime}$ values using this methods can thus be considered to be an upper limit.

The experimentally determined yield stress values from rheological experiments for aqueous CNC-salt systems (Table 2) were compared to the gel modulus at $E=1$ (Table 1) to compare their relative magnitude to the Laplace pressure. It can readily be seen that in all of the cases for $\mathrm{CNC}$ suspensions containing $\mathrm{NaCl}$, the magnitude of the yield stress under equilibrium conditions is not sufficient to resist capillary pressure, and thus these systems are not expected to inhibit capillary flow. In order to understand dynamic changes of yield stress relatively to concentration changes, we have determined the concentration changes in the evaporating droplets in-line with our previous work. ${ }^{1}$ A description of the calculation method can also be found in Supporting Information. The calculations gave an understanding of average weight concentration changes in the drop (Figure 4). This method does not allow for local concentration gradients since spatial information of the particles within the droplet is missing. If one would like to estimate local concentrations, other analysis methods such as computational fluid dynamics should be used but the accuracy will also heavily depend on the simulation assumptions. ${ }^{64}$ For our analysis using dimensionless numbers, average concentrations suffice to get a feel for order of magnitudes of opposing forces. We can thus refer to bulk concentration changes in the colloidal drops and analyze the yield stress values for specific concentration regime. For instance, when the weight concentration of $\mathrm{CNCs}$ increases to $10 \mathrm{wt} \%$ for drying colloidal drops at longer times of initially $4 \mathrm{wt} \% 10 \mathrm{mM} \mathrm{NaCl}$ (Figure 4), the recorded yield stress value $(8.52 \mathrm{~Pa})$ is still lower than the required gel modulus to stop capillary flow (Table 2). On the other hand, it gets difficult to determine the yield stress values for systems with added salt where the weight concentration of CNCs exceeds $10 \mathrm{wt} \%$ since homogenous mixing of the 
salt and gelled CNCs cannot be achieved due to aggregation. For the studied concentrations of

$\mathrm{CNC}$ suspensions, the determined yield stress values when $\mathrm{CaCl}_{2}$ is added to the suspensions reach sufficiently high values to stop capillary flow. Yield stress values for $\mathrm{CaCl}_{2}$ at longer times (past $30 \mathrm{~min}$ ) cannot be recorded due to inhomogeneous flow which is caused by the

253 formation of aggregation lumps of CNCs.

Table 1 Elastic modulus values required to attain $E=1$ for CNCs Suspensions with Added 255 Salt (10 mM).

\begin{tabular}{ccc} 
CNCs & $\mathrm{NaCl}$ & $\mathrm{CaCl}_{2}$ \\
$($ wt \%) & $G^{\prime}$ & $G^{\prime}$ \\
& $(\mathrm{Pa})$ & $(\mathrm{Pa})$ \\
\hline 2 & 50.08 & 52.87 \\
4 & 37.44 & 30.83
\end{tabular}

Table 2 Yield Stress Values for CNCs Suspensions with Added Salt (10 mM).

\begin{tabular}{|c|c|c|c|c|c|}
\hline \multirow{6}{*}{$\begin{array}{l}\text { CNCs } \\
(w t \%)\end{array}$} & $\mathrm{NaCl}$ & $\mathrm{NaCl}$ & $\mathrm{NaCl}$ & $\mathrm{CaCl}_{2}$ & $\mathrm{CaCl}_{2}$ \\
\hline & $\sigma_{\mathrm{y}}$ & $\sigma_{\mathrm{y}}$ & $\sigma_{\mathrm{y}}$ & $\sigma_{y}$ & $\sigma_{y}$ \\
\hline & $(\mathrm{Pa})$ & $(\mathrm{Pa})$ & $(\mathrm{Pa})$ & $(\mathrm{Pa})$ & $(\mathrm{Pa})$ \\
\hline & Initial & After & After & Initial & After \\
\hline & & 30 & 1 & & $30 \mathrm{~min}$ \\
\hline & & $\min$ & $\mathrm{hr}$ & & \\
\hline 2 & - & 0.31 & 0.64 & 1.02 & 23.38 \\
\hline 4 & 0.20 & 1.52 & 10.32 & 9.49 & 119.84 \\
\hline 10 & 8.52 & 22.89 & - & 74.18 & - \\
\hline
\end{tabular}

259 The modified capillary number gives us a means to describe the effect of elastic resistance opposing the capillary flow but does not take into account the effects of Marangoni stresses. 
Surface tension gradients induced by gelation will create Marangoni stress at the interface of droplets which is balanced by a shear stress within the liquid. For example, the surface tension of ethyl cellulose nanoparticle suspensions has previously been shown to decrease upon salt addition. ${ }^{65}$ This mechanism was explained in two different ways: In the first scenario, nanoparticle aggregates formed in the bulk are adsorbed at the air-water interface alongside single nanoparticles whereas in the second scenario only single nanoparticles are adsorbed at the air-water interface while nanoparticle aggregates are formed at the interface by the attachment of nanoparticles onto other already at the interface. As the adsorption is a dynamic process there will inherently be concentration gradients on the surface giving rise to local Marangoni stresses. In addition, the surface tension of evaporating nanofluid droplets have previously been analyzed using the pendant drop method. ${ }^{66}$ It was shown that an increase of nanoparticle concentration such as silver or laponite also caused a decrease in the measured surface tension values. Laponite suspensions are known to exhibit sol-gel transitions at increased concentrations, similar to CNCs at higher ionic strength. ${ }^{67,68}$ Specifically, when the laponite concentration was increased to $6 \mathrm{wt} \%$, the surface tensions of the droplet decreased from $70 \mathrm{mN} / \mathrm{m}$ to $27 \mathrm{mN} / \mathrm{m} .{ }^{66}$

The dynamic boundary condition for Marangoni flow is obtained as Equation 4 describing the fluid flow. The hydrodynamic interaction tensor can be used to evaluate the forces of particles and thus predict the forces and particle velocities. ${ }^{69,70}$

$\eta_{1} \frac{\partial u_{t 1}}{\partial n}+\eta_{1} \frac{\partial u_{n 1}}{\partial t}-\eta_{2} \frac{\partial u_{t 2}}{\partial n}-\eta_{2} \frac{\partial u_{n 2}}{\partial t}=-[\nabla \gamma-\hat{n}(\hat{n} \cdot \nabla \gamma)]$

where the right-hand side of equation describes the tangential stress caused by surface tension, $\nabla \gamma$ is the surface tension gradient, $\eta$ is the viscosity of the medium, $u_{t}$ is the velocity tangent to the interface, $u_{n}$ is the normal component, and $\hat{n}$ is the unit normal pointing out of the surface. 
Without having to fully solve Equation 4 over the drying droplet, we can analyze the effect of surface tension gradients and the viscosity of the suspensions on the deposition using the Marangoni number, $M_{a}$ (Equation 5), using the same dimensionless number approach as used for the effect of the yield stress on capillary flow. The Marangoni numbers were calculated for the $\mathrm{CNC}$ suspensions as a function of the salt concentration in line with our previous work using Equation 7. ${ }^{1}$

$M_{a}=-\frac{d \gamma}{d c} \frac{l_{c}}{\eta D_{p a r}} \Delta c$

where $d \gamma / d c$ is the surface tension change with the change of the salt concentration (Figure SI 1), $l_{c}$ is the length scale of the droplet (diameter of the droplet), $\eta$ is the viscosity of the suspension, and $D_{p a r}$ is the parallel particle diffusivity. The product of $-\left(\frac{d \gamma}{d c}\right) \Delta c$ is the magnitude of the surface tension difference across the droplet determined by the difference between the surface tension of the suspension containing salt and the blank CNC suspension. The values for the diffusion coefficients and calculated Marangoni numbers for $4 \mathrm{wt} \% \mathrm{CNC}$ suspension as function of the amount of $\mathrm{NaCl}$ added are combined in Table 3. The calculated Marangoni numbers can then be used to understand the relative magnitude of Marangoni flow with respect to the capillary flow. In this work, Marangoni stresses are induced by gradients of the surface tension in the gelled suspension due to the addition of salt into the CNC suspension. 
Nanocrystal Suspension containing $\mathrm{NaCl}$. $\mathrm{D}_{\mathrm{per}}, \mathrm{D}_{\mathrm{par}}$ and $\mathrm{D}_{\text {rot }}$ are respectively the diffusion coefficients perpendicular and parallel to the particle main axis, and the rotational diffusion coefficient around the main axis.

\begin{tabular}{c|c|c|c|c|}
\hline $\mathrm{NaCl}$ & $\begin{array}{c}\mathrm{D}_{\text {per }} \\
(\mathrm{mM})\end{array}$ & $\begin{array}{c}\mathrm{D}_{\text {par }} \\
\left(\mu \mathrm{m}^{2} / \mathrm{s}\right)\end{array}$ & $\begin{array}{c}\mathrm{D}_{\text {rot }} \\
\left(\mu \mathrm{m}^{2} / \mathrm{s}\right)\end{array}$ & $\begin{array}{c}\mathrm{M}_{\mathrm{a}} \\
\left(\mathrm{s}^{-1}\right)\end{array}$ \\
\hline 1 & 0.54 & 3.23 & 18 & 0.6 \\
\hline 5 & 0.52 & 2.7 & 24 & 0.7 \\
10 & 0.052 & 0.68 & 13 & 3.4 \\
\hline
\end{tabular}

Increased Marangoni numbers with increasing $\mathrm{NaCl}$ concentration (Table 3) indicate that Marangoni flow, i.e. the reverse particle flow toward the center of the drying droplet, becomes more important relative to capillary flow, the flow of particles toward the outer edges of the droplet due to a higher water evaporation rate at these edges. Diffusion coefficients for $\mathrm{CaCl}_{2}$ added CNC suspensions cannot be deduced with light scattering experiments because relaxations times become longer and gelation sets in within the measurement time for relevant $\mathrm{CaCl}_{2}$ concentrations.

The importance of the relative magnitude of Marangoni flow to processes inside the drying

319 droplet can further be shown by calculating the modified Péclet numbers (Equations 6-8) where $\Delta \gamma$ is the surface tension difference with added salt and blank CNC suspension, $v_{c} \sim J_{e} / l_{c}^{2}$ is the evaporation velocity, $J_{e}$ is the evaporation flux, $\eta$ is the viscosity of the suspension (Figure SI 8), $D_{p}$ is the particle diffusivity (Table 3 ), and $l_{c}$ is the length scale of the droplet: 
$325 P e_{M a / E}=\frac{\Delta \gamma}{\eta v_{c}}$

$326 P e_{M a}$ describes the relative effect of Marangoni stress to the diffusive properties of the

327 suspended particles whereas $P e_{E}$ compares capillary flow via evaporation velocity to the 328 particle diffusive properties. Both numbers thus describe the ability or inability of particle 329 diffusion to offset concentration gradients induced by macroscopic flows, i.e. Marangoni or 330 capillary flow respectively. Dividing these two dimensionless numbers results in another 331 dimensionless number $P e_{M a / E}$ used to compare the magnitude of Marangoni flow to the capillary flow in the drying droplets similarly to the earlier defined Marangoni number (Equation 5). The calculated values for $P e_{M a / E}$ numbers are given in Figure 1 for $4 \mathrm{wt} \% \mathrm{CNC}$ suspension with added salt and can be used to compare the magnitude of contributing factors in drying colloidal drops. It can clearly be seen that $P e_{M a / E}$ decreases as time progresses showing that the relative importance of Marangoni flow to capillary flow decreases during the deposition.

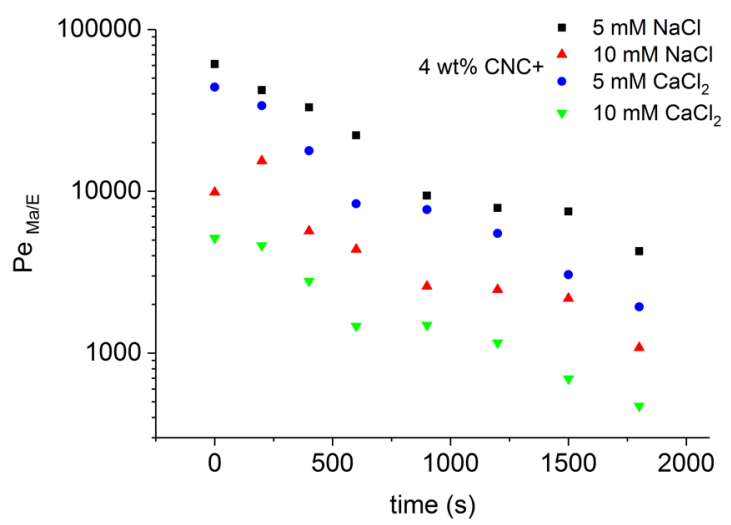


341 To investigate the kinetics of gelation of the suspensions, we carried out time sweep rheology experiments (Figure 2a, Figure SI 2, 4). For neat CNC suspensions, the viscous modulus 343 dominates the elastic modulus, an indication of viscous fluid-like behavior. On the other hand, 344 suspensions with salt showed the opposite where the elastic modulus dominated the viscous 345 modulus. With time, gelling progressed as seen by an increase in elastic modulus with time 346 (Figure 2a). In general, the modulus ultimately reaches a plateau indicating the end of the 347 gelation process (Figure SI 4). ${ }^{71}$ The suspension of CNCs mixed with salt kept overnight in 348 closed vials also showed no flow upon inversion (Figure SI 6), a further indication of gelation. 349 The no-slip boundary condition is not satisfied at longer times ( $>1$ hour) in our experiments 350 where a plateau is observed ideally for elastic modulus values in Figure 2a. The addition of $351 \mathrm{NaCl}$ resulted in gel formation displaying a frequency-independent elastic modulus (Figure 2b).

352 The slight increase in elastic modulus observed at higher frequencies was associated with 353 instrument inertia. Furthermore, a clear increase in the complex viscosity at lower frequencies 354 with the addition of salt to CNC suspension indicated the formation of a network (Figure SI 5). 355 These results are comparable to results obtained in the literature. ${ }^{49,52}$

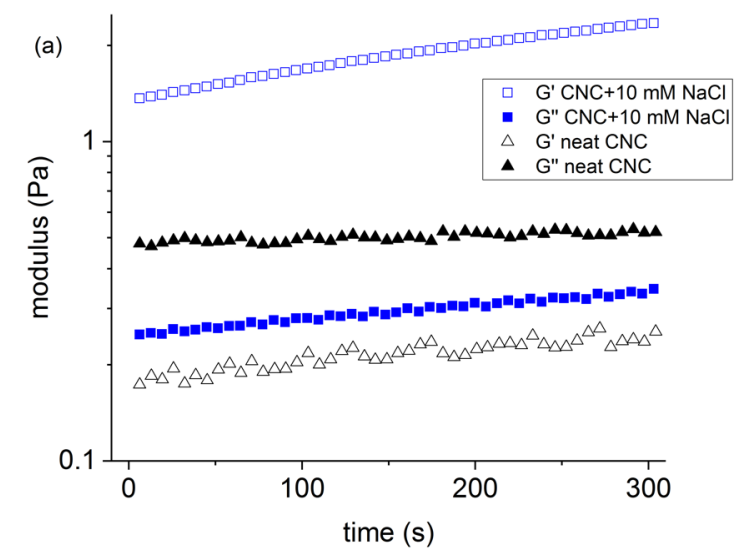




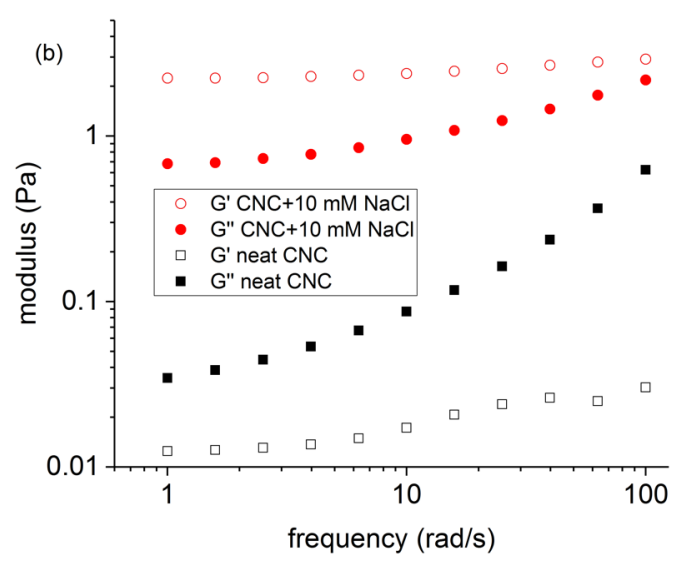

Figure 2 (a) Time sweep (b) frequency sweep measurements of $2 \mathrm{wt} \% \mathrm{CNC}$ suspensions in water and added salt (elastic modulus, open; viscous modulus, closed).

So far we have introduced the key factors which control the deposition of the colloidal films, the discussion will be followed by the analysis of the deposition patterns of the obtained films based on these factors and their importance. Firstly, the deposition at the outer edges of the drying droplets, caused by capillary flow, can be expected to decrease with increasing amounts of added salt as the increased viscosity reduces capillary flow.

In order to quantify these changes in the final deposit, we define a new parameter, the deposition number (Equation 9), relating the maximum height of the outer ring deposit, $h_{\text {max,edge, }}$ to the average film thickness at the centre of the film, $h_{\text {average,center: }}$

If the deposition number is larger than 1, the outer ring deposits, i.e. the "coffee-ring", will 371 dominate in the deposition profile, whereas if it is equal to 1, the deposit is uniform. We have plotted the deposition number versus $\mathrm{NaCl}$ concentration for different $\mathrm{CNC}$ concentrations in 373 Figure $3 \mathrm{a}$ by using the data from profilometry of the final deposits. We also compared the calculated deposition number to the ratio of maximum height of the film to minimum height of 
375 the film (Table SI 1). Similar trends were obtained for these ratios compared to obtained

376 deposition numbers as defined by equation (9) for both 4 and $2 \mathrm{wt} \% \mathrm{CNC}$ suspensions with

377 only magnitude of the values differing. A decrease is seen for $4 \mathrm{wt} \%$ as the $\mathrm{NaCl}$ content is

378 increased, whereas the values for $2 \mathrm{wt} \% \mathrm{CNC}$ suspensions remained somewhat constant.

379 However, using the average, i.e. the DN as defined by equation (9) has the advantage that it still

380 gives a usuable value for deposits which have sections without any deposition. This is seen for

$3812 \mathrm{wt} \% \mathrm{CNCs}$ with $5 \mathrm{mM} \mathrm{NaCl}$, where a central section is left empty because the drying drop

382 becomes depleted of particles at later stages of drying and the minimum height becomes 0 .
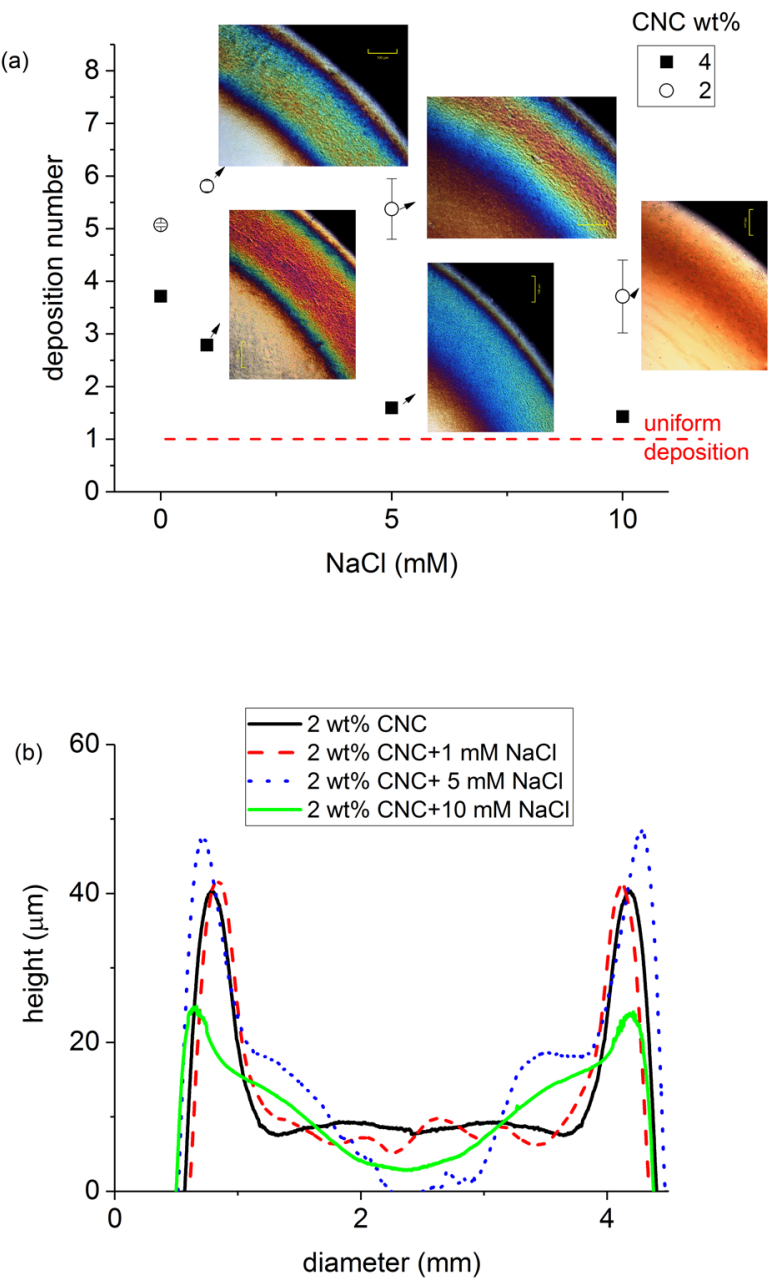

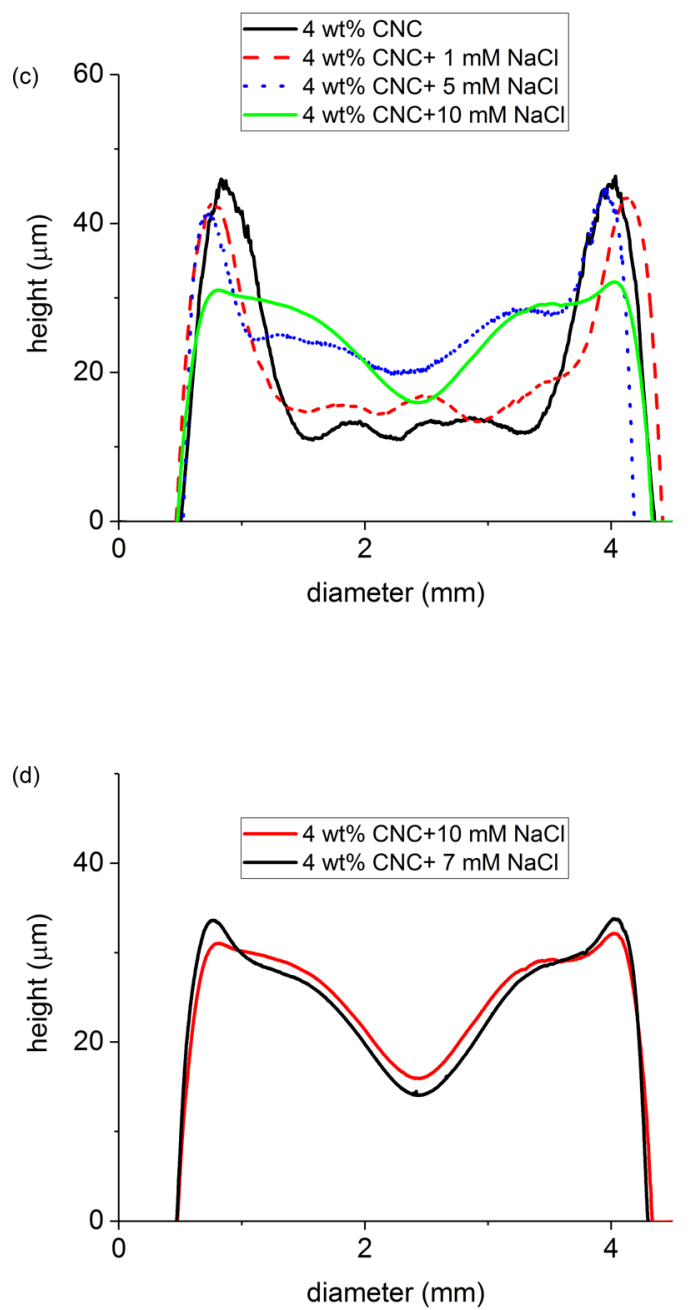

Figure 3 (a) Deposition number of films deposited from a $\mathrm{CNC}-\mathrm{NaCl}$ aqueous mixture with corresponding cross-polarized light microscope images from edge of the films, film profiles for $\mathrm{NaCl}$ addition to (b) $\mathbf{2} \mathbf{w t} \%$ CNC and (c) 4 wt\% CNC suspension and (d) comparison of change of salt concentration.

When $5 \mathrm{mM} \mathrm{NaCl}$ is added to a $2 \mathrm{wt} \% \mathrm{CNC}$ suspension, the particles form a network structure.

These structures are carried to the perimeter of the colloidal drop by capillary flow that also gives rise to the coffee-ring effect. This is not seen when $10 \mathrm{mM} \mathrm{NaCl}$ is added because the increase in the viscosity of the system retards the particle motion and limits/blocks the capillary flow. (Figure 3b). As can be seen for $2 \mathrm{wt} \% \mathrm{CNC}+10 \mathrm{mM} \mathrm{NaCl}$, the decrease in the coffeering becomes apparent and coincides with an increase in the suspension viscosity (Figure SI 2).

For the suspension of $4 \mathrm{wt} \% \mathrm{CNC}+5 \mathrm{mM} \mathrm{NaCl}$, the yield stress could not be recorded after initial mixing and the deposition of the film is controlled mostly due to Marangoni and capillary 
flows for the first $16 \mathrm{~min}$. This is seen in Figure 1 where $P e_{M a / E}$ is large and decreases during

400

401

402

403

404

405

406

407

408

409

410

411

412

413

414

drying. Although the viscosity of the suspension increases gradually with time for this system, it only becomes important in controlling the deposition profile after $\sim 16$ min (Figure 1, 4). Viscous resistance was thus found to be effective only from $\sim 16$ min to the end of deposition at around $36 \mathrm{~min}$. Intermittent concentrations of salts were not studied in detail since the change of height profiles were minor in the obtained films (Figure 3d).

The contribution of the viscosity of the suspension dominates $P e_{M a / E}$ for $4 \mathrm{wt} \% \mathrm{CNC}+10 \mathrm{mM}$ $\mathrm{NaCl}$ which results in lower values than $P e_{M a / E}$ for $4 \mathrm{wt} \% \mathrm{CNC}+5 \mathrm{mM} \mathrm{NaCl}$. The ensuing effect on the deposition is directly seen in the shape of the final deposit with a reduced height of the outer ring deposit relative to the center height (Figure 1, 3a-c). Uniform films cannot be obtained for the studied concentration regime since the measured magnitude of the yield stress of the suspension is not sufficient to fully suppress radial capillary flow (Table 3).

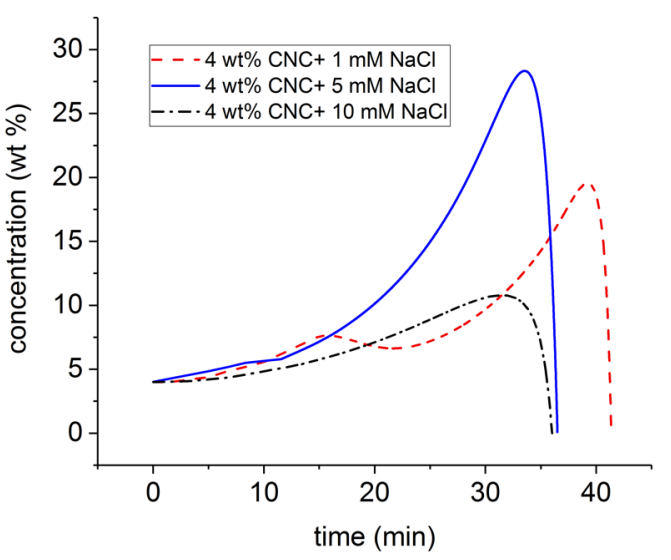

Figure 4 Average weight concentration variation of cellulose nanocrystals in the drying droplets with added $\mathrm{NaCl}$.

The observed thickness changes at the perimeter of the films were further analyzed by crosspolarized light microscopy and included in Figure 3a for direct comparison. The colored band variation were further correlated with Michel-Lévy color chart ${ }^{72,73}$ in terms of optical path 
difference (OPD) (Figure SI 13). The changes in colors could be directly related to the thickness changes as described in our earlier work and is therefore unsurprising. ${ }^{1}$ The obtained rainbow pattern is thus a direct effect of the deposit thickness at the outer edge and the slope of outer edge deposit peaks, and can thus be fully controlled by the deposition conditions.

On the other hand, small aggregates (Figure 5a, 5c) were observed for the dried films from electrostatic interactions between CNCs as measured by zeta potential determinations (SI Table

2).
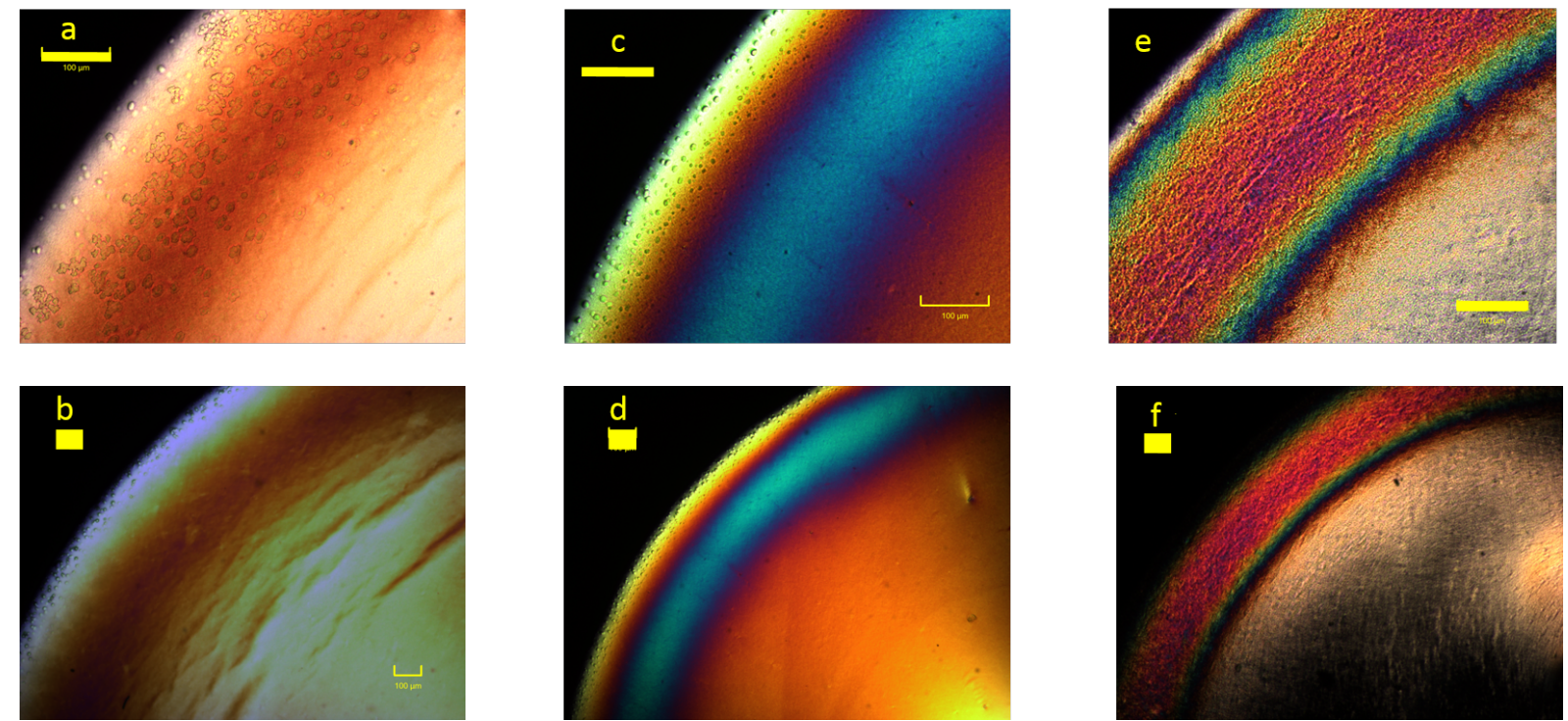

Figure 5 Cross-polarized light microscope images for salt added films (a, b) $2 \mathrm{wt} \% \mathrm{CNC}+10 \mathrm{mM} \mathrm{NaCl}(\mathrm{c}$,

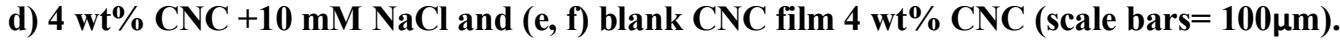

Further increasing the $\mathrm{NaCl}$ concentration to $25 \mathrm{mM}$ led to problems associated with stratification (Figure SI 14, 15). Indeed, deposits clearly showed stratification at an increased concentration of salt for $2 \mathrm{wt} \% \mathrm{CNC}+25 \mathrm{mM} \mathrm{NaCl}$ suspension (Figure SI 14). It has also been 
pointed out that the evaporation rate controls the stratification for bi-dispersed systems, ${ }^{74,75}$ and that volume fraction of particles affect stratification. ${ }^{75}$

The center of the films deposited from a $\mathrm{CNC}-\mathrm{NaCl}$ mixture still has color characterized by cross-polarized light microscopy (Figure 5, SI 17), indicating retained individualization of the nanocrystals and thus sufficient colloidal stability within the time frame of the deposition process. The observed color under cross-polarized light microscope however disappears around $15 \mathrm{mM}$ for $\mathrm{NaCl}$ added $\mathrm{CNC}$ suspensions of $2 \mathrm{wt} \%$ and $4 \mathrm{wt} \%$ (Figure SI 13). For CNC suspensions containing $\mathrm{CaCl}_{2}$, color disappears already at lower concentrations $(5 \mathrm{mM})$ for both $2 \mathrm{wt} \%$ and $4 \mathrm{wt} \%$ CNCs (Figure SI 17).

Considering color formation of the films in visible light, the pitch distance values of chiral nematic assembly must be reduced to a certain range to reflect visible light. ${ }^{9,1}$ The concentration inside the drying droplets for all $4 \mathrm{wt} \% \mathrm{CNC}$ suspension can be seen to increase as the droplet dries, going through a maximum towards the end of the drying process (Figure 4). During weight concentration changes for the suspension of initially $4 \mathrm{wt} \% \mathrm{CNC}+1 \mathrm{mM} \mathrm{NaCl}$ a peak is observed at 15 min due to the depinning stage of the drop in which some of the formed skin collapses. However, we see the average concentrations in the drying droplets reaching much higher values for suspensions containing 1 and $5 \mathrm{mM} \mathrm{NaCl}$, while the increase in concentration was suppressed when $10 \mathrm{mM} \mathrm{NaCl}$ was added to the suspension. This suppression for $10 \mathrm{mM}$ $\mathrm{NaCl}$ is either due to gelation or the deposition of the skin during drying. It has to be noted that retraction of the contact line can also reduce the particle deposition at the edge since particles only deposit within the confined drop geometry. ${ }^{76,77}$ In addition, the initial contact angle of the colloidal drops also influences the colloidal deposition process. ${ }^{28,78}$ The contact angle of water on the glass cover slides used was determined to be $62.3^{\circ} \pm 2.5^{\circ}$ whereas initial contact angle of $4 \mathrm{wt} \% \mathrm{CNC}$ colloidal drop decreased to $54.7^{\circ} \pm 2.2^{\circ}$. This contact angle decrease is thus a result of the particle loading and the reduced surface tension of the drop (Figure SI 1). For the $4 \mathrm{wt} \%$ 
$\mathrm{CNC}$ suspension with $5 \mathrm{mM} \mathrm{NaCl}$ added, the initial contact angle was recorded to be $55.2^{\circ} \pm 4.9^{\circ}$ while $4 \mathrm{wt} \%+10 \mathrm{mM} \mathrm{NaCl}$ had a contact angle of $52.2^{\circ} \pm 3.8^{\circ}$. When $\mathrm{CaCl}_{2}$ was added to 4 wt $\% \mathrm{CNC}$ suspensions, the contact angle became $46.0^{\circ} \pm 3.1^{\circ}$ for $5 \mathrm{mM} \mathrm{CaCl}_{2}$ and $36.9^{\circ} \pm 2.7^{\circ}$ for $10 \mathrm{mM} \mathrm{CaCl}_{2}$. These changes show a similar trend as the surface tension variations (Figure SI 1). One could thus expect stronger capillary flow upon salt addition (smaller gap between surface and air-water interface) but this is of course opposed by gelation induced by the same salt addition. The reduction in coffee-ring height indicates that gelation is the prevailing effect in these systems.

Clear iridescence was observed for the dried films with initially $4 \mathrm{wt} \%$ neat CNCs whereas the droplets of $4 \mathrm{wt} \% \mathrm{CNC}$ containing 1 and $5 \mathrm{mM} \mathrm{NaCl}$ did not result in iridescent films although their concentration increased during drying. This can be due to the loss of the chiral nematic structure (network formation) or because the pitch value increases at increased ionic strength via induced electrostatic interactions ${ }^{79}$ or alternatively, gelation may avoid a further decrease in the pitch length due to jamming of nanocrystals ${ }^{80}$ or kinetic arrest. It was also shown that increased ionic strength shifts the phase sequence toward the isotropic phase. ${ }^{81}$

The effect of $\mathrm{CaCl}_{2}$ addition to $\mathrm{CNC}$ suspension and film formation was also studied. The addition of $\mathrm{CaCl}_{2}$ directly leads to gelation (Figure SI 19-21). Contrary to $\mathrm{NaCl}$, films obtained by $\mathrm{CaCl}_{2}$ addition to $\mathrm{CNC}$ suspension showed a transition from a ring-shaped deposit to domeshaped deposits (Figure 6a, 6b). It was shown earlier (Table 3) that the yield stress is high enough for suspensions containing $\mathrm{CaCl}_{2}$ to stop capillary flow and this can be seen in the deposits taking on a dome-shaped form. Indeed, the outer edge deposits are the direct result of dominant capillary flow. For $4 \mathrm{wt} \% \mathrm{CNC}$ suspensions, an increase in $\mathrm{CaCl}_{2}$ concentration from $5 \mathrm{mM}$ to $10 \mathrm{mM}$ leads to a decrease in $P e_{M a / E}$ throughout the deposition (Figure 1) which indicates the dome-shaped deposit is obtained by elastic resistance i.e. an increased yield stress retarding the capillary flow rather than the induced flow due to surface tension gradients after 
gelation of the drying drop. For $4 \mathrm{wt} \% \mathrm{CNC}+5 \mathrm{mM} \mathrm{CaCl}_{2}$ the contribution of Marangoni flow is higher for the first $10 \mathrm{~min}$ than at later times of evaporation. This causes a decrease in terms of the height of the coffee-ring compared to obtained film of $4 \mathrm{wt} \% \mathrm{CNC}$ (Figure $6 \mathrm{a}, \mathrm{b}$ ). When we compare the films obtained from drying a $4 \mathrm{wt} \% \mathrm{CNC}+5 \mathrm{mM} \mathrm{CaCl}_{2}$ suspension to the film obtained from $4 \mathrm{wt} \% \mathrm{CNC}+10 \mathrm{mM} \mathrm{NaCl}$, both films showed a two-humped deposition pattern. With $5 \mathrm{mM} \mathrm{NaCl}$ the humps are of lower height at the summit than for $10 \mathrm{mM} \mathrm{NaCl}$, which can be attributed to a higher $P e_{M a / E}$ for $4 \mathrm{wt} \% \mathrm{CNC}+5 \mathrm{mM} \mathrm{CaCl}_{2}$ during the deposition (Figure 1).

Restricting capillary flow avoids the coffee-ring deposits with the typical rainbow color patterns. Deposition number cannot be calculated for systems where the outer ring is inexistent since the location of the edge cannot be defined, and the deposition number becomes infinity. In addition, films obtained by the addition of $\mathrm{CaCl}_{2}$ to $\mathrm{CNC}$ suspensions showed no color formation under cross-polarized light due to the loss of the structure formation (Figure SI 17). These mixtures led to films of flocculated CNCs which is attributed to fact that calcium ions form bridging complexes with CNCs, unlike sodium ions which only screen $\mathrm{CNC}$ charges. ${ }^{82}$ These changes can be characterized through oscillatory shear measurements by comparing the modulus values of the suspensions at the same concentration for different salts (Figure SI 9, 20, 21). In all cases, suspensions with added $\mathrm{CaCl}_{2}$ showed higher modulus values, attributed to increased percolation. This was also confirmed by changed electrostatic interactions between CNCs measured by zeta potential measurements (SI Table 2).

505 Another observation for the flocculation induced by $\mathrm{Ca}^{2+}$ ions is the formation of sharkskin patterns on the surface of the film (Figure 6a). Sharkskin occurs because induced elasticity in colloidal drop cannot compensate the flocculation related crack formation in colloidal deposit. ${ }^{83}$ 

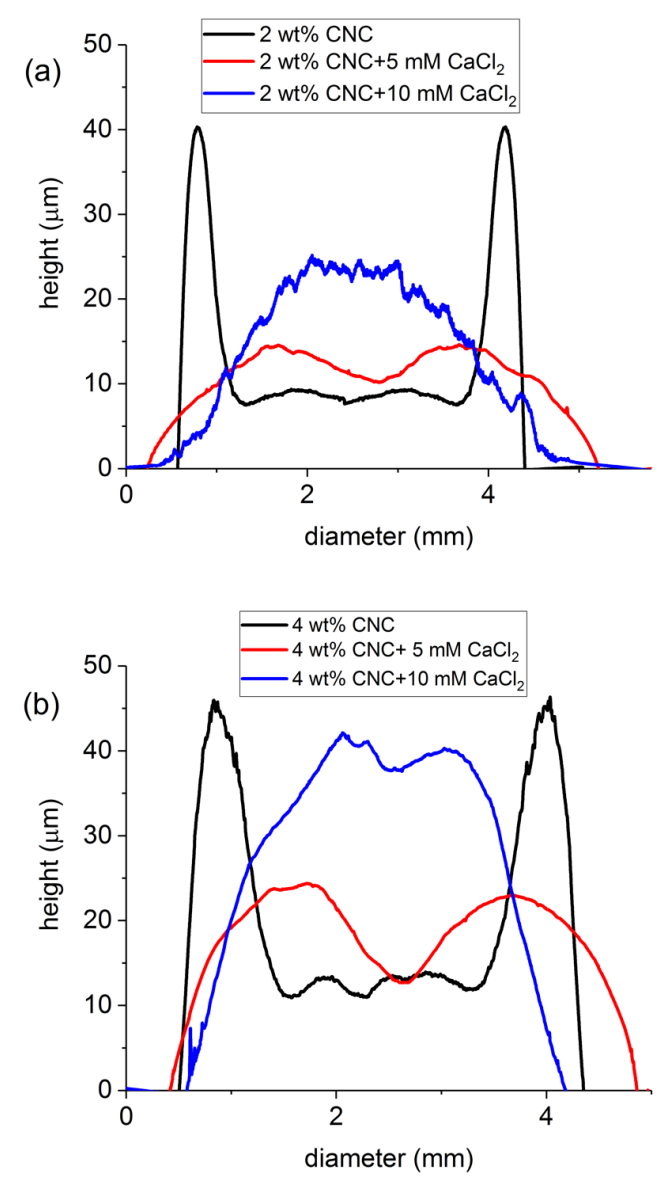

Figure 6 Film profiles for $\mathrm{CaCl}_{2}$ addition to (a) $2 \mathrm{wt} \% \mathrm{CNC}$ suspension (b) $4 \mathrm{wt} \% \mathrm{CNC}$ suspension.

Understanding the mechanism of evaporative self-assembly allows us to control the morphology

512 of the deposition of the films. We have gained an understanding of the effect of elastic stress

513 via induced gelation and Marangoni flow via changes of the surface tension on the deposition of CNC films. This method might be a good alternative to solutal or solvent-mediated induced

515 Marangoni flow techniques since the latter may cause aggregation problems. CNCs are also not compatible with all organic solvents and surfactants may introduce unwanted impurities in

517 the films.

\section{CONCLUSION}

Gelation controls the deposition of CNCs at increased salt concentrations, whereas capillary

521 flow shapes the final deposition pattern of CNCs for low salt concentrations. However, the addition of salt changes the electrostatic interactions between CNCs which causes changes in 
523

524

525

526

527

528

529

530

531

532

533

534

535

536

537

538

539

540

541

542

543

544

terms of the colloidal stability and self-assembly which is crucial to obtain optically active films. Both the addition of $\mathrm{NaCl}$ or $\mathrm{CaCl}_{2}$ induces gelation for the suspension. The addition of $\mathrm{NaCl}$ retains the structure formation for $\mathrm{CNCs}$ and thus results in optically active films via Bragg refraction, whereas the addition of $\mathrm{CaCl}_{2}$ results in loss of colloidal stability and the structure formation of CNCs. The transition from ring-shaped deposition to dome-shaped deposition occurs for $\mathrm{CaCl}_{2}$ added $\mathrm{CNC}$ suspensions due to gelation during the deposition.

\section{ACKNOWLEDGEMENTS}

The authors would like to thank Research Foundation - Flanders (FWO) for funding under the Odysseus grant (G.0C60.13N) and KU Leuven for grant OT/14/072. Authors thank to Dr. Hartmut Kriegs for the experimental support for DDLS and Dr. Christina Schütz for AFM measurement. W.T. also thanks to the Provincie West-Vlaanderen (Belgium) for financial support through his Provincial Chair in Advanced Materials.

\section{ASSOCIATED CONTENT}

Supporting Information. More characterization of CNCs; DDLS measurements, Atomic force microscopy, sulfate ester groups, rheological measurements and cross-polarized microscope images. This material is available free of charge via the Internet at http://pubs.acs.org

\section{AUTHOR INFORMATION}

Corresponding Author

wim.thielemans@kuleuven.be

Author Present Address 
545 Renewable Materials and Nanotechnology Research Group, Department of Chemical

546 Engineering, KU Leuven, Campus Kulak Kortrijk, Etienne Sabbelaan 53, 8500 Kortrijk,

547 Belgium

548 Author Contributions

549 A.G. developed the concept for the study, carried out all the experiments unless otherwise stated

550 and wrote the manuscript. W.T. supervised the study and edited the manuscript. K.K.

551 supervised DDLS measurements. A.G. and J.V.R. prepared different batches of CNCs

552 suspensions and mixed these batches. S.L. determined the concentration of sulfate ester group

553 of CNCs.

554

\section{REFERENCES}

559 1. Gençer, A.; Schütz, C.; Thielemans, W., Influence of the Particle Concentration and Marangoni Flow on the Formation of Cellulose Nanocrystal Films. Langmuir 2017, 33 (1), 228-234.

$5612 . \quad$ Stavenga, D. G., Thin Film and Multilayer Optics Cause Structural Colors of Many Insects and

562 Birds. Materials Today: Proceedings 2014, 1, 109-121.

563 3. Jacobs, M.; Lopez-Garcia, M.; Phrathep, O. P.; Lawson, T.; Oulton, R.; Whitney, H. M.,

564 Photonic multilayer structure of Begonia chloroplasts enhances photosynthetic efficiency. Nat. Plants $5652016,2,16162$.

$566 \quad 4 . \quad$ Xiao, M.; Li, Y.; Allen, M. C.; Deheyn, D. D.; Yue, X.; Zhao, J.; Gianneschi, N. C.; Shawkey, M.

567 D.; Dhinojwala, A., Bio-Inspired Structural Colors Produced via Self-Assembly of Synthetic Melanin

568 Nanoparticles. ACS Nano 2015, 9 (5), 5454-5460.

$569 \quad 5 . \quad$ Vignolini, S.; Rudall, P. J.; Rowland, A. V.; Reed, A.; Moyroud, E.; Faden, R. B.; Baumberg, J. J.;

570 Glover, B. J.; Steiner, U., Pointillist structural color in Pollia fruit. Proceedings of the National

571 Academy of Sciences 2012, 109 (39), 15712-15715.

$572 \quad 6 . \quad$ Rodríguez, R. E.; Agarwal, S. P.; An, S.; Kazyak, E.; Das, D.; Shang, W.; Skye, R.; Deng, T.;

573 Dasgupta, N. P., Biotemplated Morpho Butterfly Wings for Tunable Structurally Colored

574 Photocatalysts. ACS Appl. Mater. Interfaces 2018, 10 (5), 4614-4621.

575 7. Wilts, B. D.; Dumanli, A. G.; Middleton, R.; Vukusic, P.; Vignolini, S., Invited Article: Chiral

576 optics of helicoidal cellulose nanocrystal films. APL Photonics 2017, 2 (4), 040801. 
577 8. Dumanli, A. G.; van der Kooij, H. M.; Kamita, G.; Reisner, E.; Baumberg, J. J.; Steiner, U.; 578 Vignolini, S., Digital Color in Cellulose Nanocrystal Films. ACS Appl. Mater. Interfaces 2014, 6 (15), $579 \quad 12302-12306$.

580 9. Mu, X.; Gray, D. G., Formation of Chiral Nematic Films from Cellulose Nanocrystal 581 Suspensions Is a Two-Stage Process. Langmuir 2014, 30 (31), 9256-9260.

582 10. Lagerwall, J. P. F.; Schütz, C.; Salajkova, M.; Noh, J.; Hyun Park, J.; Scalia, G.; Bergström, L., 583 Cellulose nanocrystal-based materials: from liquid crystal self-assembly and glass formation to multifunctional thin films. NPG Asia Mater. 2014, 6, e80.

11. Parker, R. M.; Frka-Petesic, B.; Guidetti, G.; Kamita, G.; Consani, G.; Abell, C.; Vignolini, S., Hierarchical Self-Assembly of Cellulose Nanocrystals in a Confined Geometry. ACS Nano 2016, 10 (9), 8443-8449.

12. Gu, M.; Jiang, C.; Liu, D.; Prempeh, N.; Smalyukh, I. I., Cellulose Nanocrystal/Poly(ethylene glycol) Composite as an Iridescent Coating on Polymer Substrates: Structure-Color and Interface Adhesion. ACS Appl. Mater. Interfaces 2016, 8 (47), 32565-32573.

13. Wang, B.; Walther, A., Self-Assembled, Iridescent, Crustacean-Mimetic Nanocomposites with Tailored Periodicity and Layered Cuticular Structure. ACS Nano 2015, 9 (11), 10637-10646.

14. Bardet, R.; Belgacem, N.; Bras, J., Flexibility and Color Monitoring of Cellulose Nanocrystal Iridescent Solid Films Using Anionic or Neutral Polymers. ACS Appl. Mater. Interfaces 2015, 7 (7), 4010-4018.

15. Yao, K.; Meng, Q.; Bulone, V.; Zhou, Q., Flexible and Responsive Chiral Nematic Cellulose Nanocrystal/Poly(ethylene glycol) Composite Films with Uniform and Tunable Structural Color. Advanced Materials 2017, 29 (28), 1701323.

16. Araki, J.; Kuga, S., Effect of Trace Electrolyte on Liquid Crystal Type of Cellulose Microcrystals. Langmuir 2001, 17 (15), 4493-4496.

17. Pan, J.; Hamad, W.; Straus, S. K., Parameters Affecting the Chiral Nematic Phase of Nanocrystalline Cellulose Films. Macromolecules 2010, 43 (8), 3851-3858.

18. Oguzlu, H.; Danumah, C.; Boluk, Y., Colloidal behavior of aqueous cellulose nanocrystal suspensions. Curr. Opin. Colloid Interface Sci. 2017, 29, 46-56.

19. Cherhal, F.; Cousin, F.; Capron, I., Influence of Charge Density and Ionic Strength on the Aggregation Process of Cellulose Nanocrystals in Aqueous Suspension, as Revealed by Small-Angle Neutron Scattering. Langmuir 2015, 31 (20), 5596-5602.

20. Chau, M.; Sriskandha, S. E.; Pichugin, D.; Thérien-Aubin, H.; Nykypanchuk, D.; Chauve, G.; Méthot, M.; Bouchard, J.; Gang, O.; Kumacheva, E., Ion-Mediated Gelation of Aqueous Suspensions of Cellulose Nanocrystals. Biomacromolecules 2015, 16 (8), 2455-2462.

21. De France, K. J.; Hoare, T.; Cranston, E. D., Review of Hydrogels and Aerogels Containing Nanocellulose. Chem. Mater. 2017, 29 (11), 4609-4631.

22. Xu, Y.; Atrens, A. D.; Stokes, J. R., "Liquid, gel and soft glass" phase transitions and rheology of nanocrystalline cellulose suspensions as a function of concentration and salinity. Soft Matter 2018, 14 (10), 1953-1963.

23. Phan-Xuan, T.; Thuresson, A.; Skepö, M.; Labrador, A.; Bordes, R.; Matic, A., Aggregation behavior of aqueous cellulose nanocrystals: the effect of inorganic salts. Cellulose 2016, 23 (6), 36533663.

24. Yang, J.; Xu, F.; Han, C.-R., Metal Ion Mediated Cellulose Nanofibrils Transient Network in Covalently Cross-linked Hydrogels: Mechanistic Insight into Morphology and Dynamics. Biomacromolecules 2017, 18 (3), 1019-1028.

25. Peddireddy, K. R.; Capron, I.; Nicolai, T.; Benyahia, L., Gelation Kinetics and Network Structure of Cellulose Nanocrystals in Aqueous Solution. Biomacromolecules 2016, 17 (10), 32983304.

26. Bigioni, T. P.; Lin, X.-M.; Nguyen, T. T.; Corwin, E. I.; Witten, T. A.; Jaeger, H. M., Kinetically driven self assembly of highly ordered nanoparticle monolayers. Nat. Mater. 2006, 5 (4), 265-270. 27. Boles, M. A.; Engel, M.; Talapin, D. V., Self-Assembly of Colloidal Nanocrystals: From Intricate Structures to Functional Materials. Chem. Rev. 2016, 116 (18), 11220-11289. 
28. Davidson, Z. S.; Huang, Y.; Gross, A.; Martinez, A.; Still, T.; Zhou, C.; Collings, P. J.; Kamien, R. D.; Yodh, A. G., Deposition and drying dynamics of liquid crystal droplets. Nat. Commun. 2017, 8, 15642.

29. Daly, R.; Kotova, O.; Boese, M.; Gunnlaugsson, T.; Boland, J. J., Chemical Nano-Gardens: Growth of Salt Nanowires from Supramolecular Self-Assembly Gels. ACS Nano 2013, 7 (6), 48384845.

30. Deegan, R. D.; Bakajin, O.; Dupont, T. F.; Huber, G.; Nagel, S. R.; Witten, T. A., Capillary flow as the cause of ring stains from dried liquid drops. Nature 1997, 389 (6653), 827-829.

31. Hu, H.; Larson, R. G., Analysis of the Effects of Marangoni Stresses on the Microflow in an Evaporating Sessile Droplet. Langmuir 2005, 21 (9), 3972-3980.

32. Still, T.; Yunker, P. J.; Yodh, A. G., Surfactant-Induced Marangoni Eddies Alter the CoffeeRings of Evaporating Colloidal Drops. Langmuir 2012, 28 (11), 4984-4988.

33. Seo, C.; Jang, D.; Chae, J.; Shin, S., Altering the coffee-ring effect by adding a surfactant-like viscous polymer solution. Sci. Rep. 2017, 7 (1), 500.

34. Kim, H.; Muller, K.; Shardt, O.; Afkhami, S.; Stone, H. A., Solutal Marangoni flows of miscible liquids drive transport without surface contamination. Nat. Phys. 2017, 13, 1105.

35. Varanakkottu, S. N.; Anyfantakis, M.; Morel, M.; Rudiuk, S.; Baigl, D., Light-Directed Particle Patterning by Evaporative Optical Marangoni Assembly. Nano Lett. 2016, 16 (1), 644-650.

36. Dugyala, V. R.; Basavaraj, M. G., Control over Coffee-Ring Formation in Evaporating Liquid Drops Containing Ellipsoids. Langmuir 2014, 30 (29), 8680-8686.

37. Bhardwaj, R.; Fang, X.; Somasundaran, P.; Attinger, D., Self-Assembly of Colloidal Particles from Evaporating Droplets: Role of DLVO Interactions and Proposition of a Phase Diagram. Langmuir 2010, 26 (11), 7833-7842.

38. Anyfantakis, M.; Baigl, D., Dynamic Photocontrol of the Coffee-Ring Effect with Optically Tunable Particle Stickiness. Angew. Chem., Int. Ed. 2014, 53 (51), 14077-14081.

39. Li, Y.; Yang, Q.; Li, M.; Song, Y., Rate-dependent interface capture beyond the coffee-ring effect. Sci. Rep. 2016, 6, 24628.

40. Talbot, E. L.; Yang, L.; Berson, A.; Bain, C. D., Control of the Particle Distribution in Inkjet Printing through an Evaporation-Driven Sol-Gel Transition. ACS Appl. Mater. Interfaces 2014, 6 (12), 9572-9583.

41. Buskens, P.; Burghoorn, M.; Mourad, M. C. D.; Vroon, Z., Antireflective Coatings for Glass and Transparent Polymers. Langmuir 2016, 32 (27), 6781-6793.

42. Biswas, P. K., Sol-gel thin films for optics and photonics. J. Sol-Gel Sci. Technol 2011, 59 (3), 456-474.

43. Håkansson, K. M. O.; Fall, A. B.; Lundell, F.; Yu, S.; Krywka, C.; Roth, S. V.; Santoro, G.; Kvick, M.; Prahl Wittberg, L.; Wågberg, L.; Söderberg, L. D., Hydrodynamic alignment and assembly of nanofibrils resulting in strong cellulose filaments. Nat. Commun. 2014, 5, 4018.

44. Siqueira, G.; Kokkinis, D.; Libanori, R.; Hausmann, M. K.; Gladman, A. S.; Neels, A.; Tingaut, P.; Zimmermann, T.; Lewis, J. A.; Studart, A. R., Cellulose Nanocrystal Inks for 3D Printing of Textured Cellular Architectures. Adv. Funct. Mater. 2017, 27 (12), 1604619.

45. Palaganas, N. B.; Mangadlao, J. D.; de Leon, A. C. C.; Palaganas, J. O.; Pangilinan, K. D.; Lee, Y. J.; Advincula, R. C., 3D Printing of Photocurable Cellulose Nanocrystal Composite for Fabrication of Complex Architectures via Stereolithography. ACS Appl. Mater. Interfaces 2017, 9 (39), 34314-34324. 46. Li, V. C.-F.; Dunn, C. K.; Zhang, Z.; Deng, Y.; Qi, H. J., Direct Ink Write (DIW) 3D Printed Cellulose Nanocrystal Aerogel Structures. Sci. Rep. 2017, 7 (1), 8018.

47. Berry, J. D.; Neeson, M. J.; Dagastine, R. R.; Chan, D. Y. C.; Tabor, R. F., Measurement of surface and interfacial tension using pendant drop tensiometry. J. Colloid Interface Sci. 2015, 454, 226-237.

48. Nagel, M.; Tervoort, T. A.; Vermant, J., From drop-shape analysis to stress-fitting elastometry. Adv. Colloid Interface Sci. 2017, 247 (Supplement C), 33-51.

49. Lenfant, G.; Heuzey, M.-C.; van de Ven, T. G. M.; Carreau, P. J., A comparative study of ECNC and CNC suspensions: effect of salt on rheological properties. Rheol. Acta 2017, 56 (1), 51-62. 
50. Frisken, B. J., Revisiting the method of cumulants for the analysis of dynamic light-scattering data. Appl. Opt. 2001, 40 (24), 4087-4091.

51. Lehner, D.; Lindner, H.; Glatter, O., Determination of the Translational and Rotational Diffusion Coefficients of Rodlike Particles Using Depolarized Dynamic Light Scattering. Langmuir 2000, 16 (4), 1689-1695.

52. Bertsch, P.; Isabettini, S.; Fischer, P., Ion-Induced Hydrogel Formation and Nematic Ordering of Nanocrystalline Cellulose Suspensions. Biomacromolecules 2017, 18 (12), 4060-4066.

53. Dong, X. M.; Kimura, T.; Revol, J.-F.; Gray, D. G., Effects of lonic Strength on the Isotropic-Chiral Nematic Phase Transition of Suspensions of Cellulose Crystallites. Langmuir 1996, 12 (8), 2076-2082.

54. Lyubimova, O.; Stoyanov, S. R.; Gusarov, S.; Kovalenko, A., Electric Interfacial Layer of Modified Cellulose Nanocrystals in Aqueous Electrolyte Solution: Predictions by the Molecular Theory of Solvation. Langmuir 2015, 31 (25), 7106-7116.

55. Shafiei-Sabet, S.; Hamad, W. Y.; Hatzikiriakos, S. G., lonic strength effects on the microstructure and shear rheology of cellulose nanocrystal suspensions. Cellulose 2014, 21 (5), 33473359.

56. Rajneesh, B.; Xiaohua, F.; Daniel, A., Pattern formation during the evaporation of a colloidal nanoliter drop: a numerical and experimental study. New J. Phys. 2009, 11 (7), 075020.

57. Hwang, H.; Son, G., Numerical Simulation of Evaporation-Induced Particle Line Formation on a Moving Substrate. Heat Transfer Eng. 2017, 1-7.

58. Okuzono, T.; Kobayashi, M.; Doi, M., Final shape of a drying thin film. Physical review. E, Statistical, nonlinear, and soft matter physics 2009, 80 (2 Pt 1), 021603.

59. Hu, H.; Larson, R. G., Marangoni Effect Reverses Coffee-Ring Depositions. J. Phys. Chem. $B$ 2006, 110 (14), 7090-7094.

60. Chen, P.; Harmand, S.; Ouenzerfi, S.; Schiffler, J., Marangoni Flow Induced Evaporation Enhancement on Binary Sessile Drops. J. Phys. Chem. B 2017, 121 (23), 5824-5834.

61. Kaplan, C. N.; Mahadevan, L., Evaporation-driven ring and film deposition from colloidal droplets. J. Fluid Mech. 2015, 781, R2.

62. Sempels, W.; De Dier, R.; Mizuno, H.; Hofkens, J.; Vermant, J., Auto-production of biosurfactants reverses the coffee ring effect in a bacterial system. Nat. Commun. 2013, 4, 1757. 63. Marculescu, C.; Tincu, B.; Avram, A.; Burinaru, T.; Avram, M., Computational Prediction of Capillary Number Impact on Droplets Formation in Microchannels. Energy Procedia 2016, 85 (Supplement C), 339-349.

64. Gruber, M. F.; Johnson, C. J.; Tang, C. Y.; Jensen, M. H.; Yde, L.; Hélix-Nielsen, C., Computational fluid dynamics simulations of flow and concentration polarization in forward osmosis membrane systems. J. Membr. Sci. 2011, 379 (1), 488-495.

65. Bizmark, N.; loannidis, M. A., Effects of lonic Strength on the Colloidal Stability and Interfacial Assembly of Hydrophobic Ethyl Cellulose Nanoparticles. Langmuir 2015, 31 (34), 9282-9289.

66. Chen, R.-H.; Phuoc, T. X.; Martello, D., Surface tension of evaporating nanofluid droplets. Int. J. Heat Mass Transfer 2011, 54 (11), 2459-2466.

67. Cummins, H. Z., Liquid, glass, gel: The phases of colloidal Laponite. J. Non-Cryst. Solids 2007, 353 (41), 3891-3905.

68. Jatav, S.; Joshi, Y. M., Phase Behavior of Aqueous Suspension of Laponite: New Insights with Microscopic Evidence. Langmuir 2017, 33 (9), 2370-2377.

69. Lisicki, M.; Nägele, G., Colloidal Hydrodynamics and Interfacial Effects. In Soft Matter at Aqueous Interfaces, Lang, P.; Liu, Y., Eds. Springer International Publishing: Cham, 2016; pp 313-386. 70. Kirby, B. J., Chapter 1: Kinematics, conservation equations, and boundary conditions. In Micro- and Nanoscale Fluid Mechanics: Transport in Microfluidic Devices, Press, C. U., Ed. Cambridge University Press: New York, 2010.

71. Zuidema, J. M.; Rivet, C. J.; Gilbert, R. J.; Morrison, F. A., A protocol for rheological characterization of hydrogels for tissue engineering strategies. J. Biomed. Mater. Res., Part B 2014, $102(5), 1063-1073$. 
72. Stoiber, R. E.; Morse, S. A., Anisotropic crystals. In Crystal Identification with the Polarizing Microscope, Hall, C., Ed. Springer US: 2012; pp 102-115.

73. Wayne, R., Interference Microscopy. In Light and Video Microscopy, Academic Press/Elsevier: 2009; pp 149-166.

74. Howard, M. P.; Nikoubashman, A.; Panagiotopoulos, A. Z., Stratification Dynamics in Drying Colloidal Mixtures. Langmuir 2017, 33 (15), 3685-3693.

75. Trueman, R. E.; Lago Domingues, E.; Emmett, S. N.; Murray, M. W.; Keddie, J. L.; Routh, A. F., Autostratification in Drying Colloidal Dispersions: Experimental Investigations. Langmuir 2012, 28 (7), 3420-3428.

76. Morales, V. L.; Parlange, J.-Y.; Wu, M.; Pérez-Reche, F. J.; Zhang, W.; Sang, W.; Steenhuis, T. S., Surfactant-Mediated Control of Colloid Pattern Assembly and Attachment Strength in Evaporating Droplets. Langmuir 2013, 29 (6), 1831-1840.

77. Anyfantakis, M.; Baigl, D.; Binks, B. P., Evaporation of Drops Containing Silica Nanoparticles of Varying Hydrophobicities: Exploiting Particle-Particle Interactions for Additive-Free Tunable Deposit Morphology. Langmuir 2017, 33 (20), 5025-5036.

78. Boulogne, F.; Ingremeau, F.; Limat, L.; Stone, H. A., Tuning the Receding Contact Angle on Hydrogels by Addition of Particles. Langmuir 2016, 32 (22), 5573-5579.

79. Hirai, A.; Inui, O.; Horii, F.; Tsuji, M., Phase Separation Behavior in Aqueous Suspensions of Bacterial Cellulose Nanocrystals Prepared by Sulfuric Acid Treatment. Langmuir 2009, 25 (1), 497502.

80. Kang, K.; Dhont, J. K. G., Glass Transition in Suspensions of Charged Rods: Structural Arrest and Texture Dynamics. Phys. Rev. Lett. 2013, 110 (1), 015901.

81. Honorato-Rios, C.; Kuhnhold, A.; Bruckner, J. R.; Dannert, R.; Schilling, T.; Lagerwall, J. P. F., Equilibrium Liquid Crystal Phase Diagrams and Detection of Kinetic Arrest in Cellulose Nanocrystal Suspensions. Frontiers in Materials 2016, 3 (21).

82. Gebauer, D.; Oliynyk, V.; Salajkova, M.; Sort, J.; Zhou, Q.; Bergstrom, L.; Salazar-Alvarez, G., A transparent hybrid of nanocrystalline cellulose and amorphous calcium carbonate nanoparticles. Nanoscale 2011, 3 (9), 3563-3566.

83. Singh, K. B.; Bhosale, L. R.; Tirumkudulu, M. S., Cracking in Drying Colloidal Films of Flocculated Dispersions. Langmuir 2009, 25 (8), 4284-4287. 
772

773

774

775

776

777

778

779

780

781

782

TOC Graphic

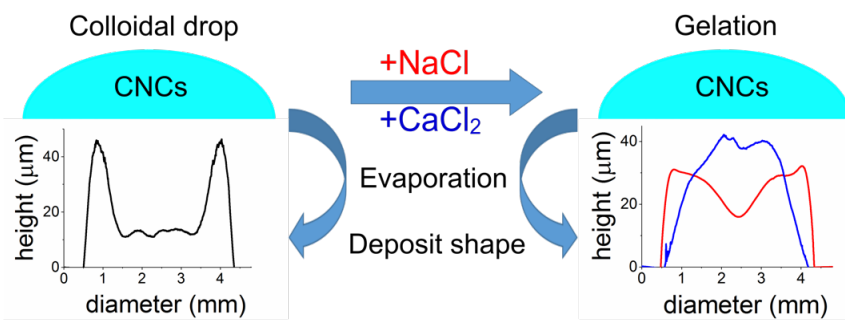

783

784 